\title{
Totally Contact Umbilical Lightlike Hypersurfaces of Indefinite $\mathcal{S}$-Manifolds
}

\author{
Rachna Rani, ${ }^{1}$ Rakesh Kumar, ${ }^{2}$ and R. K. Nagaich ${ }^{3}$ \\ ${ }^{1}$ University College, Moonak, Punjab 148033, India \\ ${ }^{2}$ University College of Engineering, Punjabi University, Patiala 147002, India \\ ${ }^{3}$ Department of Mathematics, Punjabi University, Patiala 147002, India \\ Correspondence should be addressed to Rakesh Kumar; dr_rk37c@yahoo.co.in
}

Received 22 August 2012; Revised 5 November 2012; Accepted 8 November 2012

Academic Editor: Mingsheng Liu

Copyright (C) 2013 Rachna Rani et al. This is an open access article distributed under the Creative Commons Attribution License, which permits unrestricted use, distribution, and reproduction in any medium, provided the original work is properly cited.

We study totally contact umbilical lightlike hypersurfaces of indefinite $\mathcal{S}$-manifolds and prove the nonexistence of totally contact umbilical lightlike hypersurface in indefinite $\mathcal{S}$-space form.

\section{Introduction}

The general theory of nondegenerate submanifolds of Riemannian or semi-Riemannian manifolds is one of the most important topics of differential geometry $[1,2]$. But the theory of degenerate or lightlike submanifolds of semiRiemannian manifolds is relatively new [3] and different due to the fact that their normal vector bundle intersects with the tangent bundle. Thus, the study of lightlike submanifolds becomes more difficult than the study of nondegenerate submanifolds and one cannot use, in the usual way, the classical theory of submanifold to define induced objects on a lightlike submanifold. The degenerate geometry rises within the semi-Riemannian context, due to the existence of causal character of geometrical objects: their spacelike, timelike, or lightlike nature implies the existence of three types of hypersurfaces and submanifolds.

A lightlike framed hypersurface of a Lorentz $C$-manifold, with an induced metric connection, is a Killing horizon and a globally hyperbolic spacetime; a de Sitter spacetime can carry a framed structure $[3,4]$. Moreover, the contact geometry has a significant use in differential equations, optics, and phase spaces of dynamical systems [5-7]. All these motivated us to study lightlike hypersurfaces of indefinite globally framed $f$ manifold, in particular indefinite $\delta$-manifolds.

In this paper, we study totally contact umbilical lightlike hypersurfaces of indefinite $\mathcal{S}$-manifolds and prove the nonexistence of totally contact umbilical lightlike hypersurface in indefinite $\delta$-space form.

\section{Preliminaries}

A manifold $\bar{M}$ of dimension $2 n+r$ is called a globally framed $f$-manifold (g.f.f-manifold) if it is endowed with a nowhere vanishing $(1,1)$-tensor field $\bar{\phi}$ of constant rank, such that $\operatorname{ker} \bar{\phi}$ is parallelizable; that is, there exist global vector fields $\bar{\zeta}_{\alpha}, \alpha \in$ $\{1, \ldots, r\}$, with their dual 1 -forms $\bar{\eta}^{\alpha}$, satisfying

$$
\bar{\phi}^{2}=-I+\bar{\eta}^{\alpha} \otimes \bar{\zeta}_{\alpha}, \quad \bar{\eta}^{\alpha}\left(\bar{\zeta}_{\beta}\right)=\delta_{\beta}^{\alpha}
$$

If the metric $\bar{g}$ is a semi-Riemannian metric with index $v$, $0<v<2 n+r$ such that

$$
\bar{g}(\bar{\phi} X, \bar{\phi} Y)=\bar{g}(X, Y)-\sum_{\alpha=1}^{r} \epsilon_{\alpha} \bar{\eta}^{\alpha}(X) \bar{\eta}^{\alpha}(Y),
$$

for any $X, Y \in \Gamma(T \bar{M}), \epsilon_{\alpha}= \pm 1$ accordingly $\bar{\zeta}_{\alpha}$ is either spacelike or timelike, then the g.f.f-manifold $\left(\bar{M}^{2 n+r}, \bar{\phi}, \bar{\zeta}_{\alpha}, \bar{\eta}^{\alpha}\right)$ is called an indefinite metric $g . f . f$-manifold. Then clearly $\bar{\eta}^{\alpha}(X)=\epsilon_{\alpha} \bar{g}\left(X, \bar{\zeta}_{\alpha}\right)$, for any $\alpha \in\{1, \ldots, r\}$. An indefinite metric g.f. $f$-manifold is called an indefinite $\mathcal{S}$-manifold if it is normal that is, the tensor field $N=N_{\bar{\phi}}+2 d \bar{\eta}^{\alpha} \otimes \bar{\zeta}_{\alpha}$ 
vanishes, where $N_{\bar{\phi}}$ is Nijenhuis tensor of $\bar{\phi}$ and $d \bar{\eta}^{\alpha}=\Phi$, for any $\alpha \in\{1, \ldots, r\}$, where $\Phi(X, Y)=\bar{g}(X, \bar{\phi} Y)$, for any $X, Y \in \Gamma(T \bar{M})$. The Levi-Civita connection of an indefinite $\mathcal{S}$-manifold satisfies

$$
\left(\bar{\nabla}_{X} \bar{\phi}\right) Y=\bar{g}(\bar{\phi} X, \bar{\phi} Y) \bar{\zeta}+\bar{\eta}(Y) \bar{\phi}^{2}(X),
$$

where $\bar{\zeta}=\sum_{\alpha=1}^{r} \bar{\zeta}_{\alpha}$ and $\bar{\eta}=\sum_{\alpha=1}^{r} \epsilon_{\alpha} \bar{\eta}^{\alpha}$. Also

$$
\bar{\nabla}_{X} \bar{\zeta}_{\alpha}=-\epsilon_{\alpha} \bar{\phi} X
$$

and $\operatorname{ker} \bar{\phi}$ is integrable flat distribution, since $\bar{\nabla}_{\bar{\zeta}_{\alpha}} \bar{\zeta}_{\beta}=0$, for detail see [8].

We recall notations and fundamental equations for lightlike hypersurfaces, which are due to Duggal and Bejancu [3].

Let $(\bar{M}, \bar{g})$ be a $(2 n+1)$-dimensional semi-Riemannian manifold with index $s, 0<s<2 n+1$, and let $(M, g)$ be a hypersurface of $\bar{M}$, with $g=\left.g\right|_{M}$. $M$ is a lightlike hypersurface of $\bar{M}$ if $g$ is of constant rank $2 n-1$ and the normal bundle $T M^{\perp}$ is a distribution of rank 1 on $M$. A complementary bundle of $T M^{\perp}$ in $T M$ is a rank $2 n-1$ nondegenerate distribution over $M$. It is denoted by $S(T M)$ and known as a screen distribution. Therefore we have

$$
\begin{aligned}
T M & =S(T M) \perp T M^{\perp}, \\
\left.T \bar{M}\right|_{M} & =S(T M) \perp S(T M)^{\perp},
\end{aligned}
$$

where $S(T M)^{\perp}$ is a orthogonal complementary vector bundle of $S(T M)$ in $\left.T \bar{M}\right|_{M}$. The following theorem has important roles in studying the geometry of lightlike hypersurface.

Theorem 1. Let $(M, g, S(T M))$ be a lightlike hypersurface of a semi-Riemannian manifold $(\bar{M}, \bar{g})$. Then there exists a unique vector bundle $\operatorname{tr}(T M)$ of rank 1 over $M$, such that for any nonzero section $\xi$ of $T M^{\perp}$ on a coordinate neighborhood $\mathcal{U} \subset$ $M$, there exists a unique section $N$ of $\operatorname{tr}(T M)$ on $\mathcal{U}$ satisfying

$$
\begin{aligned}
\bar{g}(\xi, N)=1, \quad \bar{g}(N, N) & =\bar{g}(N, W)=0, \\
\forall W & \in \Gamma\left(\left.S(T M)\right|_{\mathscr{U}}\right) .
\end{aligned}
$$

Hence, we have the following decompositions of $\left.T \bar{M}\right|_{M}$ :

$$
\begin{aligned}
\left.T \bar{M}\right|_{M} & =S(T M) \perp\left(T M^{\perp} \oplus \operatorname{tr}(T M)\right) \\
& =T M \oplus \operatorname{tr}(T M) .
\end{aligned}
$$

Let $\bar{\nabla}$ be the Levi-Civita connection on $\bar{M}$ with respect to $\bar{g}$. Then using the decompositions in (7), Gauss and Weingarten formulae are given as

$$
\begin{aligned}
& \bar{\nabla}_{X} Y=\nabla_{X} Y+h(X, Y), \\
& \bar{\nabla}_{X} N=-A_{N} X+\nabla_{X}^{t} N,
\end{aligned}
$$

for any $X, Y \in \Gamma(T M)$ and $N \in \Gamma(\operatorname{tr}(T M))$, where $\nabla_{X} Y$ and $A_{N} X$ belong to $\Gamma(T M)$ while $h(X, Y)$ and $\nabla_{X}^{t} N$ belong to $\Gamma(\operatorname{tr}(T M))$. Here $\nabla$ is a torsion free linear connection on $M, h$ is a $\Gamma(\operatorname{tr}(T M))$-valued symmetric bilinear form on $\Gamma(T M)$ and known as the second fundamental form. $A_{N}$ is a linear operator on $\Gamma(T M)$ and known as the shape operator of lightlike immersion and $\nabla^{t}$ is a linear connection on $\Gamma(\operatorname{tr}(T M))$.

Locally, for the pair $\{\xi, N\}$, following the Duggal and Bejancu's notation [3], we recall local second fundamental form $B$ and 1 -form $\tau$ as

$$
\begin{aligned}
& B(X, Y)=\bar{g}(h(X, Y), \xi), \\
& \text { therefore } h(X, Y)=B(X, Y) N . \\
& \tau(X)=\bar{g}\left(\nabla_{X}^{t} N, \xi\right) \\
& \text { therefore } \nabla_{X}^{t} N=\tau(X) N .
\end{aligned}
$$

Hence locally, (8) becomes

$$
\begin{aligned}
& \bar{\nabla}_{X} Y=\nabla_{X} Y+B(X, Y) N, \\
& \bar{\nabla}_{X} N=-A_{N} X+\tau(X) N .
\end{aligned}
$$

If $P$ denotes the projection morphism of TM on $S(T M)$ then from (5), we have

$$
\begin{gathered}
\nabla_{X} P Y=\nabla_{X}^{*} P Y+h^{*}(X, P Y), \\
\nabla_{X} \xi=-A_{\xi}^{*} X+\nabla_{X}^{* t} \xi,
\end{gathered}
$$

for any $X, Y \in \Gamma(T M), \xi \in \Gamma\left(T M^{\perp}\right)$, where $\nabla_{X}^{*} P Y$ and $A_{\xi}^{*} X$ belong to $\Gamma(S(T M))$ while $h^{*}(X, P Y)$ and $\nabla_{X}^{* t} \xi$ belong to $\Gamma\left(T M^{\perp}\right)$. Here $h^{*}$ and $A_{\xi}^{*}$ are called the second fundamental form and the shape operator of the screen distribution, respectively. It should be noted that the induced linear connection $\nabla$ is not a metric connection as it satisfies

$$
\left(\nabla_{X} g\right)(Y, Z)=B(X, Y) \theta(Z)+B(X, Z) \theta(Y),
$$

where $\theta$ is a differential 1 form locally defined on $M$ by $\theta(\cdot)=$ $\bar{g}(N, \cdot)$. By direct calculation, we have

$$
\begin{array}{cc}
g\left(A_{N} Y, P X\right)=\bar{g}\left(N, h^{*}(Y, P X)\right), & \bar{g}\left(A_{N} Y, N\right)=0, \\
g\left(A_{\xi}^{*} X, P Y\right)=\bar{g}(\xi, h(X, P Y)), & \bar{g}\left(A_{\xi}^{*} X, N\right)=0,
\end{array}
$$

for any $X, Y \in \Gamma(T M), \xi \in \Gamma\left(T M^{\perp}\right)$, and $N \in \Gamma(\operatorname{tr}(T M))$. According to Duggal and Bejancu's notation [3], locally we have know

$$
\begin{aligned}
& C(X, P Y)=\bar{g}\left(h^{*}(X, P Y), N\right) \\
& \text { therefore } h^{*}(X, P Y)=C(X, P Y) \xi, \\
& \epsilon(X)=\bar{g}\left(\nabla_{X}^{* t} \xi, N\right) \\
& \text { therefore } \nabla_{X}^{* t} \xi=\epsilon(X) \xi .
\end{aligned}
$$

Then (11) become

$$
\begin{gathered}
\nabla_{X} P Y=\nabla_{X}^{*} P Y+C(X, P Y) \xi \\
\nabla_{X} \xi=-A_{\xi}^{*} X+\epsilon(X) \xi=-A_{\xi}^{*} X-\tau(X) \xi
\end{gathered}
$$


and also give

$$
\begin{array}{cc}
g\left(A_{N} Y, P W\right)=C(Y, P W), & \bar{g}\left(A_{N} Y, N\right)=0, \\
g\left(A_{\xi}^{*} X, P Y\right)=B(X, P Y), & \bar{g}\left(A_{\xi}^{*} X, N\right)=0 .
\end{array}
$$

From (9), it is clear that

$$
B(X, \xi)=0
$$

that is, the second fundamental form of a lightlike hypersurface is degenerate. Following the notations of Duggal and Bejancu [3], for the curvature tensor $\bar{R}$ of $\bar{M}$, we have $\bar{R}(X, Y, Z, W)=\bar{g}(\bar{R}(X, Y) Z, W)$, for any $X, Y, Z, W \in \Gamma(T \bar{M})$.

\section{Lightlike Hypersurface of Indefinite $\mathcal{S}$-Manifolds}

Let $(M, g, S(T M))$ be a lightlike hypersurface of an indefinite g.f.f-manifold $\left(\bar{M}^{2 n+r}, \bar{\phi}, \bar{\zeta}_{\alpha}, \bar{\eta}^{\alpha}, \bar{g}\right)$, and $\alpha \in\{1, \ldots, r\}$ such that the characteristic vector fields $\bar{\zeta}_{\alpha}, \alpha \in\{1, \ldots, r\}$ are tangent to $M$. As the ambient manifold $\bar{M}$ has an additional geometric structure $\bar{\phi}$, we must look for a particular screen distribution on $M$. Since $\bar{g}(\bar{\phi} \xi, \xi)=0$, for any $\xi \in \Gamma\left(T M^{\perp}\right)$ therefore $\bar{\phi}\left(T M^{\perp}\right)$ is a distribution on $M$ of rank 1 such that $T M^{\perp} \cap \bar{\phi}\left(T M^{\perp}\right)=\{0\}$. Moreover $\bar{g}(\bar{\phi} N, \xi)=-\bar{g}(N, \bar{\phi} \xi)=0$, for any $N \in \Gamma(\operatorname{tr}(T M))$, therefore $\bar{\phi} N$ is tangent to $M$. Since $\bar{g}(\bar{\phi} N, N)=0$; that is, the component of $\bar{\phi} N$ with respect to $\xi$ vanishes; this implies $\bar{\phi} N \in \Gamma(S(T M))$. As $\xi$ and $N$ are null vector fields satisfying $\bar{g}(\xi, N)=1$, therefore from (2), we deduce that $\bar{\phi} \xi$ and $\bar{\phi} N$ are null vector fields satisfying $\bar{g}(\bar{\phi} \xi, \bar{\phi} N)=1$. Hence $\bar{\phi}\left(T M^{\perp}\right) \oplus \bar{\phi}(\operatorname{tr}(T M))$ is a vector subbundle of $S(T M)$ of rank 2 . It is known [9] that if structure vector fields $\bar{\zeta}_{\alpha}, \alpha \in\{1 \ldots, r\}$ are tangent to $M$ then $\bar{\zeta}_{\alpha}$ belong to $S(T M)$. Therefore $\bar{g}\left(\bar{\phi} \xi, \bar{\zeta}_{\alpha}\right)=\bar{g}\left(\bar{\phi} N, \bar{\zeta}_{\alpha}\right)=0$ implies that there exists a nondegenerate distribution $D_{0}$ of rank $2 n-4$ on $M$ such that

$$
S(T M)=\left\{\bar{\phi}(\operatorname{tr}(T M)) \oplus \bar{\phi} T M^{\perp}\right\} \perp D_{0} \perp\left\{\bar{\zeta}_{\alpha}\right\}
$$

therefore

$$
T M=\left\{\bar{\phi}(\operatorname{tr}(T M)) \oplus \bar{\phi} T M^{\perp}\right\} \perp D_{0} \perp\left\{\bar{\zeta}_{\alpha}\right\} \perp T M^{\perp}
$$

Let $D=T M^{\perp} \perp \bar{\phi} T M^{\perp} \perp D_{0}$, and let $D^{\prime}=\bar{\phi} \operatorname{tr}(T M)$ then $D$ clearly is invariant and $D^{\prime}$ is anti-invariant under $\bar{\phi}$, and we have

$$
T M=D^{\prime} \oplus D \perp\left\{\bar{\zeta}_{\alpha}\right\} .
$$

Consider the local lightlike vector fields as

$$
\begin{gathered}
U=-\bar{\phi} N \in \bar{\phi}(\operatorname{tr}(T M))=D^{\prime}, \\
V=-\bar{\phi} \xi \in D .
\end{gathered}
$$

Using the decompositions in (21), any $X \in \Gamma(T M)$ can be written as

$$
X=S X+Q X+\sum_{\alpha=1}^{r} \bar{\eta}^{\alpha}(X) \bar{\zeta}_{\alpha}
$$

where $S$ and $Q$ are the projection morphisms of $T M$ into $D$ and $D^{\prime}$, respectively, and where $Q X=u(X) U$, and $u$ is a local 1 -form on $M$ defined by $u(X)=\bar{g}(V, X)$. Therefore

$$
u(U)=1, \quad u(X)=0, \quad \forall X \in \Gamma(D), \quad \bar{\phi}^{2} N=-N .
$$

Applying $\bar{\phi}$ to (23) and then using (24), we obtain $\bar{\phi} X=$ $\bar{\phi}(S X)+u(X) N$. By assuming $\bar{\phi}(S X)=\phi X$ for any $X \in$ $\Gamma(T M)$, we obtain a tensor field $\phi$ of type $(1,1)$ on $M$ and given by

$$
\bar{\phi} X=\phi X+u(X) N
$$

Applying $\bar{\phi}$ to (25) and then using the definition of $\phi X$, we obtain

$$
\phi^{2} X=-X+u(X) U+\sum_{\alpha=1}^{r} \bar{\eta}^{\alpha}(X) \bar{\zeta}_{\alpha} .
$$

Moreover, since $\phi X=\bar{\phi}(S X)$ therefore

$$
\phi U=0, \quad \bar{\eta}^{\alpha} \circ \phi=0, \quad u(\phi X)=0,
$$

for any $X \in \Gamma(T M)$. Thus we have the following theorem analogous to a theorem proved in [10].

Theorem 2. Let $\left(\bar{M}, \bar{\phi}, \bar{\zeta}_{\alpha}, \bar{\eta}^{\alpha}, \bar{g}\right)$ be an indefinite g.f.fmanifold and let $(M, g, S(T M))$ be a lightlike hypersurface of $\bar{M}$; then $\left(M, \phi, \bar{\zeta}_{\alpha}, U, \bar{\eta}^{\alpha}, u\right)$ is also a $g . f . f$-manifold.

Since $\phi U=0$ therefore applying $\phi$ to (26), we get $\phi^{3}+\phi=$ 0 , this implies that $\phi$ is an $f$ structure of constant rank on $M$. Now using (2) and (25), we obtain

$$
\begin{aligned}
\bar{g}(\phi X, \phi Y)= & \bar{g}(X, Y)-u(X) v(Y) \\
& -u(Y) v(X)-\sum_{\alpha=1}^{r} \epsilon_{\alpha} \bar{\eta}^{\alpha}(X) \bar{\eta}^{\alpha}(Y),
\end{aligned}
$$

for any $X, Y \in \Gamma(T \bar{M})$, where $v$ is a 1 form locally defined on $M$ by $v(\cdot)=\bar{g}(U, \cdot)$. Replace $X$ by $\phi X$ in (28) and using (26) and (27), we derive

$$
\begin{aligned}
\bar{g} & (\phi X, Y)+\bar{g}(X, \phi Y) \\
& =-u(X) \theta(Y)-u(Y) \theta(X) .
\end{aligned}
$$

Let $M$ be a lightlike hypersurface of an indefinite $\mathcal{S}$ manifold $\bar{M}$; then by using (4), (10), and (25), we obtain

$$
\nabla_{X} \bar{\zeta}_{\alpha}+B\left(X, \bar{\zeta}_{\alpha}\right) N=-\epsilon_{\alpha} \phi X-\epsilon_{\alpha} u(X) N
$$


for any $X \in \Gamma(T \bar{M})$ then comparing the tangential and transversal components, we get

$$
\begin{array}{r}
\nabla_{X} \bar{\zeta}_{\alpha}=-\epsilon_{\alpha} \phi X, \\
B\left(X, \bar{\zeta}_{\alpha}\right)=-\epsilon_{\alpha} u(X),
\end{array}
$$

this implies $h\left(X, \bar{\zeta}_{\alpha}\right)=-\epsilon_{\alpha} u(X) N$.

Using (15) and (31), we get $\nabla_{X}^{*} \bar{\zeta}_{\alpha}+C\left(X, \bar{\zeta}_{\alpha}\right) \xi=-\epsilon_{\alpha} \phi X$, taking inner product with $N \in \Gamma(\operatorname{tr}(T M))$, we get $C\left(X, \bar{\zeta}_{\alpha}\right)=$ $-\epsilon_{\alpha} \bar{g}(\phi X, N)$, and then using (29), we obtain

$$
\begin{aligned}
C\left(X, \bar{\zeta}_{\alpha}\right) & =\epsilon_{\alpha} g(X, \phi N)+u(X) \theta(N)-u(N) \theta(X) \\
& =-\epsilon_{\alpha} \bar{g}(X, U) \\
& =-\epsilon_{\alpha} v(X) .
\end{aligned}
$$

Using (3), for any $N \in \Gamma(\operatorname{tr}(T M))$, we get $\bar{\nabla}_{X} \bar{\phi} N=\bar{\phi} \bar{\nabla}_{X} N+$ $\bar{g}(X, N) \bar{\zeta}$, therefore

$$
\begin{aligned}
B(X, U) & =\bar{g}(h(X, U), \xi) \\
& =-\bar{g}\left(\bar{\nabla}_{X} \bar{\phi} N, \xi\right) \\
& =\bar{g}\left(\bar{\nabla}_{X}, \phi \xi\right) \\
& =g\left(A_{N} X, V\right) \\
& =C(X, V) .
\end{aligned}
$$

\section{Totally Contact Umbilical Lightlike Hypersurface of Indefinite $\mathcal{S}$-Manifolds}

Here we will follow Bejancu's definition [11] of totally contact umbilical submanifolds of Sasakian manifolds to state totally contact umbilical lightlike hypersurfaces of indefinite $\mathcal{S}$ manifolds.

Definition 3. A lightlike hypersurface $M$ of an indefinite $\mathcal{S}$-manifold is said to be totally contact umbilical lightlike hypersurface if the second fundamental form $h$ of $M$ satisfies

$$
\begin{aligned}
h(X, Y)= & \left\{g(X, Y)-\sum_{\alpha=1}^{r} \epsilon_{\alpha} \bar{\eta}^{\alpha}(X) \bar{\eta}^{\alpha}(Y)\right\} H \\
& +\sum_{\alpha=1}^{r} \bar{\eta}^{\alpha}(X) h\left(Y, \bar{\zeta}_{\alpha}\right) \\
& +\sum_{\alpha=1}^{r} \bar{\eta}^{\alpha}(Y) h\left(X, \bar{\zeta}_{\alpha}\right),
\end{aligned}
$$

for any $X, Y \in \Gamma(T M)$, where $H$ is a transversal vector field on $M$; that is, $H=\lambda N$, where $\lambda$ is a smooth function on $\mathcal{U} \subset M$.

Remark 4. We can also write (35) as

$$
\begin{aligned}
h(X, Y) & =B(X, Y) N \\
& =\left\{B_{1}(X, Y)+B_{2}(X, Y)\right\} N,
\end{aligned}
$$

where

$$
B_{1}(X, Y)=\lambda\left\{g(X, Y)-\sum_{\alpha=1}^{r} \epsilon_{\alpha} \bar{\eta}^{\alpha}(X) \bar{\eta}^{\alpha}(Y)\right\},
$$

and using (32)

$$
B_{2}(X, Y)=-\bar{\eta}(X) u(Y)-\bar{\eta}(Y) u(X),
$$

for any $X, Y \in \Gamma(T M)$. If $\lambda=0$, that is, $B_{1}=0$; then lightlike hypersurface $M$ is said to be totally contact geodesic.

Let $\bar{R}$ be the curvature tensor fields of $\bar{M}$; then for an indefinite $\mathcal{S}$-space form $\bar{M}(c)$, we have (see [8])

$$
\begin{gathered}
\bar{R}(X, Y, W, Z) \\
=\frac{c+3 \epsilon}{4}\{\bar{g}(\bar{\phi} Y, \bar{\phi} Z) \bar{g}(\bar{\phi} X, \bar{\phi} W) \\
\quad-\bar{g}(\bar{\phi} X, \bar{\phi} Z) \bar{g}(\bar{\phi} Y, \bar{\phi} W)\} \\
+\frac{c-\epsilon}{4}\{\Phi(W, X) \Phi(Z, Y)-\Phi(Z, X) \Phi(W, Y) \\
+2 \Phi(X, Y) \Phi(W, Z)\} \\
+\{\bar{\eta}(W) \bar{\eta}(X) \bar{g}(\bar{\phi} Z, \bar{\phi} Y)-\bar{\eta}(W) \bar{\eta}(Y) \bar{g}(\bar{\phi} Z, \bar{\phi} X) \\
+\bar{\eta}(Y) \bar{\eta}(Z) \bar{g}(\bar{\phi} W, \bar{\phi} X) \\
-\bar{\eta}(Z) \bar{\eta}(X) \bar{g}(\bar{\phi} W, \bar{\phi} Y)\},
\end{gathered}
$$

for any $X, Y, Z, W \in \Gamma(T \bar{M})$, where $\Phi(X, Y)=\bar{g}(X, \bar{\phi} Y)$ and $\epsilon=\sum_{\alpha=1}^{r} \epsilon_{\alpha}$. Using (39), we obtain

$$
\begin{aligned}
\bar{R}(X, Y, W, \xi)=\frac{c-\epsilon}{4}\{ & \bar{g}(V, X) \bar{g}(W, \bar{\phi} Y) \\
& -\bar{g}(V, Y) \bar{g}(W, \bar{\phi} X) \\
& +2 \bar{g}(V, W) \bar{g}(X, \bar{\phi} Y)\}
\end{aligned}
$$

Also, for the pair $\{\xi, N\}$ on $\mathcal{U} \subset M$, from (3.8) of page no. 94 of [3], we have

$$
\begin{aligned}
\bar{R}(X, Y, W, \xi)= & \left(\nabla_{X} B\right)(Y, W)-\left(\nabla_{Y} B\right)(X, W) \\
& +\tau(X) B(Y, W)-\tau(Y) B(X, W),
\end{aligned}
$$

$X, Y, W \in \Gamma(T M)$ and $\xi \in \Gamma\left(T M^{\perp}\right)$, where

$$
\begin{aligned}
\left(\nabla_{X} B\right)(Y, W)= & X \cdot B(Y, W)-B\left(\nabla_{X} Y, W\right) \\
& -B\left(Y, \nabla_{X} W\right) .
\end{aligned}
$$


Hence using (40) and (41), we obtain

$$
\begin{aligned}
& \left(\nabla_{X} B\right)(Y, W)-\left(\nabla_{Y} B\right)(X, W) \\
& =\frac{c-\epsilon}{4}\{\bar{g}(V, X) \bar{g}(W, \bar{\phi} Y)-\bar{g}(V, Y) \bar{g}(W, \bar{\phi} X) \\
& \quad+2 \bar{g}(V, W) \bar{g}(X, \bar{\phi} Y)\} \\
& \quad-\tau(X) B(Y, W)+\tau(Y) B(X, W),
\end{aligned}
$$

for any $X, Y, W \in \Gamma(T \bar{M})$.

Lemma 5. Let $(M, g)$ be a totally contact umbilical lightlike hypersurface of an indefinite $\mathcal{S}$-manifold $(\bar{M}, \bar{g})$; then for any $X, Y, W \in \Gamma(T M)$, one has

$$
\begin{aligned}
\left(\nabla_{X} B_{1}\right)(Y, W)= & (X \cdot \lambda)\left\{g(Y, W)-\sum_{\alpha=1}^{r} \epsilon_{\alpha} \bar{\eta}^{\alpha}(Y) \bar{\eta}^{\alpha}(W)\right\} \\
& +\lambda\{B(X, Y) \theta(W)+B(X, W) \theta(Y)\} \\
& +\lambda \bar{\eta}(W)\{\bar{g}(\phi X, Y)+u(X) \theta(Y)\} \\
& +\lambda \bar{\eta}(Y)\{\bar{g}(\phi X, W)+u(X) \theta(W)\} . \\
\left(\nabla_{X} B_{2}\right)(Y, W)= & \epsilon u(W)\{\bar{g}(Y, \phi X)+u(X) \theta(Y)\} \\
& +\epsilon u(Y)\{\bar{g}(W, \phi X)+u(X) \theta(W)\} \\
& +\bar{\eta}(W)\{B(X, \phi Y)+\tau(X) u(Y)\} \\
& +\bar{\eta}(Y)\{B(X, \phi W)+\tau(X) u(W)\} .
\end{aligned}
$$

Proof. By virtue of (37) and (42), we obtain

$$
\begin{aligned}
&\left(\nabla_{X} B_{1}\right)(Y, W)=(X \cdot \lambda)\left\{g(Y, W)-\sum_{\alpha=1}^{r} \epsilon_{\alpha} \bar{\eta}^{\alpha}(Y) \bar{\eta}^{\alpha}(W)\right\} \\
&-\lambda\left\{g\left(\nabla_{X} Y, W\right)+g\left(Y, \nabla_{X} W\right)\right\} \\
&+\lambda \sum_{\alpha=1}^{r} \epsilon_{\alpha}\left\{\bar{\eta}^{\alpha}\left(\nabla_{X} Y\right) \bar{\eta}^{\alpha}(W)\right. \\
&\left.+\bar{\eta}^{\alpha}\left(\nabla_{X} W\right) \bar{\eta}^{\alpha}(Y)\right\} .
\end{aligned}
$$

Now using (12) in (45), we have

$$
\begin{aligned}
\left(\nabla_{X} B_{1}\right)(Y, W)=(X \cdot \lambda)\left\{g(Y, W)-\sum_{\alpha=1}^{r} \epsilon_{\alpha} \bar{\eta}^{\alpha}(Y) \bar{\eta}^{\alpha}(W)\right\} \\
-\lambda\{X(g(Y, W))-B(X, Y) \theta(W) \\
-B(X, W) \theta(Y)\} \\
+\lambda \sum_{\alpha=1}^{r}\left\{g\left(\nabla_{X} Y, \bar{\zeta}_{\alpha}\right) \bar{\eta}^{\alpha}(W)\right. \\
\left.+g\left(\nabla_{X} W, \bar{\zeta}_{\alpha}\right) \bar{\eta}^{\alpha}(Y)\right\} .
\end{aligned}
$$

Again using (12), we have

$$
\begin{aligned}
g\left(\nabla_{X} Y, \bar{\zeta}_{\alpha}\right)= & X\left(g\left(Y, \bar{\zeta}_{\alpha}\right)\right)+\epsilon_{\alpha} g(Y, \phi X) \\
& +\epsilon_{\alpha} u(X) \theta(Y) .
\end{aligned}
$$

Thus from (46) and (47), the first expression of the theorem follows. Next, using (38) and (42), we obtain

$$
\begin{gathered}
\left(\nabla_{X} B_{2}\right)(Y, W)=\sum_{\alpha=1}^{r} \epsilon_{\alpha}\left\{\bar{\eta}^{\alpha}\left(\nabla_{X} Y\right) u(W)+\bar{\eta}^{\alpha}\left(\nabla_{X} W\right) u(Y)\right. \\
+\bar{\eta}^{\alpha}(W) u\left(\nabla_{X} Y\right) \\
\left.+\bar{\eta}^{\alpha}(Y) u\left(\nabla_{X} W\right)\right\} .
\end{gathered}
$$

Using (31) and (32), we get

$$
\bar{\eta}^{\alpha}\left(\nabla_{X} Y\right)=g(Y, \phi X)+u(X) \theta(Y)
$$

and from (2), (3), (10), (16), (17), (18), and (25), we obtain

$$
u\left(\nabla_{X} Y\right)=B(X, \phi Y)+\tau(X) u(Y) .
$$

Thus by substituting (49) and (50) in (48), we obtain (44).

Theorem 6. Let $\bar{M}(c)$ be an indefinite $\mathcal{S}$-space form and $M$ be a totally contact umbilical lightlike hypersurface of $\bar{M}(c)$. Then $c=-3 \epsilon$, where $\epsilon=\sum_{\alpha=1}^{r} \epsilon_{\alpha}$ and $\lambda$ satisfies the following differential equations:

$$
\begin{aligned}
& \xi \cdot \lambda+\lambda \tau(\xi)-\lambda^{2}=0, \\
& P X \cdot \lambda+\lambda \tau(P X)=0,
\end{aligned}
$$

for any $X \in \Gamma(T M)$.

Proof. Let $M$ be a totally contact umbilical lightlike hypersurface of an indefinite $\mathcal{S}$-space form $\bar{M}(c)$ of constant $\bar{\phi}$ sectional curvature $c$. Then using (35), we have $B(X, \phi Y)=$ $\lambda \bar{g}(X, \phi Y)$. Substituting (29), (44) in (43), we obtain

$$
\begin{aligned}
(X \cdot \lambda)\left\{\bar{g}(Y, W)-\sum_{\alpha=1}^{r} \epsilon_{\alpha} \bar{\eta}^{\alpha}(Y) \bar{\eta}^{\alpha}(W)\right\} \\
-(Y \cdot \lambda)\left\{\bar{g}(X, W)-\sum_{\alpha=1}^{r} \epsilon_{\alpha} \bar{\eta}^{\alpha}(X) \bar{\eta}^{\alpha}(W)\right\} \\
+\lambda\{B(X, W) \theta(Y)-B(Y, W) \theta(X)\} \\
+2 \lambda\{\bar{g}(\phi X, Y)+u(X) \theta(Y)\} \bar{\eta}(W) \\
+\lambda\{(\bar{g}(W, \phi X) \bar{\eta}(Y)-\bar{g}(W, \phi Y) \bar{\eta}(X)\} \\
+\lambda\{u(X) \bar{\eta}(Y)-u(Y) \bar{\eta}(X)\} \theta(W) \\
+2 \epsilon\{\bar{g}(\phi X, Y)+u(X) \theta(Y)\} u(W) \\
+\{\lambda \bar{g}(X, \phi Y)+\tau(X) u(Y) \\
+\lambda \bar{g}(Y, \phi X)-\tau(Y) u(X)\} \bar{\eta}(W)
\end{aligned}
$$




$$
\begin{gathered}
+\lambda\{\bar{g}(X, \phi W) \bar{\eta}(Y)-\bar{g}(Y, \phi W) \bar{\eta}(X)\} \\
+\{\tau(X) \bar{\eta}(Y)-\tau(Y) \bar{\eta}(X)\} u(W) \\
+\epsilon\{\bar{g}(W, \phi X) u(Y)-\bar{g}(W, \phi Y) u(X)\} \\
=\frac{c-\epsilon}{4}\{\bar{g}(V, X) \bar{g}(W, \bar{\phi} Y) \\
-\bar{g}(V, Y) \bar{g}(W, \bar{\phi} X) \\
+2 \bar{g}(V, W) \bar{g}(X, \bar{\phi} Y)\} \\
+\tau(Y) B(X, W)-\tau(X) B(Y, W),
\end{gathered}
$$

for any $X, Y, W \in \Gamma(T \bar{M})$. Put $X=\xi$ in (52); we get

$$
\begin{aligned}
(\xi \cdot \lambda)\left\{\bar{g}(Y, W)-\sum_{\alpha=1}^{r} \epsilon_{\alpha} \bar{\eta}^{\alpha}(Y) \bar{\eta}^{\alpha}(W)\right\} \\
-\lambda B(Y, W)-2 \lambda u(Y) \bar{\eta}(W) \\
-\lambda u(W) \bar{\eta}(Y)-2 \epsilon u(Y) u(W) \\
+\tau(\xi) u(Y) \bar{\eta}(W)+2 \lambda u(Y) \bar{\eta}(W) \\
+\tau(\xi) u(W) \bar{\eta}(Y)-\epsilon u(W) u(Y) \\
+\lambda u(W) \bar{\eta}(Y) \\
=\frac{c-\epsilon}{4}\{u(Y) u(W)+2 u(W) u(Y)\} \\
\quad-\tau(\xi) B(Y, W) .
\end{aligned}
$$

Put $Y=W=U$ in (53) and then using $g(U, U)=0, u(U)=$ $1, \bar{\eta}^{\alpha}(U)=0$; we obtain

$$
-\lambda B(U, U)-3 \epsilon=3 \frac{(c-\epsilon)}{4}-\tau(\xi) B(U, U),
$$

since $B(U, U)=0$, we get $c=-3 \epsilon$. Moreover, by putting $Y=$ $V$ and $W=U$ in (52), we obtain

$$
\xi \cdot \lambda+\lambda \tau(\xi)-\lambda^{2}=0
$$

Finally, putting $X=P X, Y=P Y$, and $W=P W$ in (52) with $c=-3 \epsilon$ and using that $S(T M)$ is nondegenerate, we obtain

$$
\begin{aligned}
& \{P X \cdot \lambda+\lambda \tau(P X)\}\left(P Y-\sum_{\alpha=1}^{r} \bar{\eta}^{\alpha}(P Y) \bar{\zeta}_{\alpha}\right) \\
& =\{P Y \cdot \lambda+\lambda \tau(P Y)\}\left(P X-\sum_{\alpha=1}^{r} \bar{\eta}^{\alpha}(P X) \bar{\zeta}_{\alpha}\right) .
\end{aligned}
$$

Putting $P X=\bar{\zeta}_{\alpha}$ in (56), we get

$$
\left\{\bar{\zeta}_{\alpha} \cdot \lambda+\lambda \tau\left(\bar{\zeta}_{\alpha}\right)\right\}\left(P Y-\sum_{\alpha=1}^{r} \bar{\eta}^{\alpha}(P Y) \bar{\zeta}_{\alpha}\right)=0 .
$$

By taking $Y=V$, we obtain

$$
\bar{\zeta}_{\alpha} \cdot \lambda+\lambda \tau\left(\bar{\zeta}_{\alpha}\right)=0
$$

Since $P X \in \Gamma(S(T M))$ therefore using (19), we can write

$$
\begin{aligned}
P X= & u(P X) U+v(P X) V+\sum_{i=1}^{2 n-4} \beta_{i} F_{i} \\
& +\sum_{\alpha=1}^{r} \epsilon_{\alpha} \bar{\eta}^{\alpha}(P X) \bar{\zeta}_{\alpha} \\
= & P X^{\prime}+\sum_{\alpha=1}^{r} \epsilon_{\alpha} \bar{\eta}^{\alpha}(P X) \bar{\zeta}_{\alpha},
\end{aligned}
$$

where $\left\{F_{i}\right\}_{1 \leq i \leq 2 n-4}$ is an orthogonal basis of $D_{0}$, then using (58), we have

$$
\begin{aligned}
P X \cdot \lambda+\lambda \tau(P X)= & \left(P X^{\prime}+\sum_{\alpha=1}^{r} \epsilon_{\alpha} \bar{\eta}^{\alpha}(P X) \bar{\zeta}_{\alpha}\right) \cdot \lambda \\
& +\lambda \tau\left(P X^{\prime}+\sum_{\alpha=1}^{r} \epsilon_{\alpha} \bar{\eta}^{\alpha}(P X) \bar{\zeta}_{\alpha}\right) \\
= & P X^{\prime} \cdot \lambda+\lambda \tau\left(P X^{\prime}\right),
\end{aligned}
$$

which leads to get from (56)

$$
\left\{P X^{\prime} \cdot \lambda+\lambda \tau\left(P X^{\prime}\right)\right\} P Y^{\prime}=\left\{P Y^{\prime} \cdot \lambda+\lambda \tau\left(P Y^{\prime}\right)\right\} P X^{\prime} .
$$

Now, assume that there exists a vector field $X_{0}$ on some neighborhood of $M$ such that $P X_{0}^{\prime} \cdot \lambda+\lambda \tau\left(P X_{0}^{\prime}\right) \neq 0$ at some point $p$ in the neighborhood. Then from (61), it is clear that all the vectors of the fiber $\left(S(T M)-\left\{\bar{\zeta}_{\alpha}\right\}\right)_{p}$ are collinear with $\left(P X_{0}^{\prime}\right)_{p}$. This contradicts $\operatorname{dim}\left(S(T M)-\left\{\bar{\zeta}_{\alpha}\right\}\right)>1$. This implies the result.

From the previous mentioned theorem, we have the following corollary.

Corollary 7. There exist no totally contact umbilical lightlike real hypersurfaces of indefinite $\mathcal{S}$-space form $\bar{M}(c)(c \neq-3 \epsilon)$ with $\bar{\zeta}_{\alpha} \in T M$.

\section{Acknowledgment}

The authors would like to thank the anonymous referee for his/her valuable suggestions that helped them to improve this paper.

\section{References}

[1] B. Y. Chen, Geometry of Submanifolds, Marcel Dekker, New York, USA, 1973.

[2] B. O'Neill, Semi-Riemannian Geometry with Applications to Relativity, Academic Press, 1983.

[3] K. L. Duggal and A. Bejancu, Lightlike Submanifolds of SemiRiemannian Manifolds and Applications, Kluwer Academic Publishers, Dordrecht, The Netherlands, 1996. 
[4] K. L. Duggal and B. Sahin, "Lightlike submanifolds of indefinite Sasakian manifolds," International Journal of Mathematics and Mathematical Sciences, vol. 2007, Article ID 57585, 21 pages, 2007.

[5] V. I. Arnold, "Contact geometry: the geometrical method of Gibbss thermodynamics," in Proceedings of the Gibbs Symposium, pp. 163-179, American Mathematical Society, New Haven, Conn, USA, 1989.

[6] S. Maclane, Geometrical Mechanics II, Lecture Notes, University of Chicago, Chicago, Ill, USA, 1968.

[7] V. E. Nazaikinskii, V. E. Shatalov, and B. Y. Sternin, Contact Geometry and Linear Differential Equations, vol. 6 of De Gruyter Expositions in Mathematics, Walter de Gruyter, Berlin, Germany, 1992.

[8] L. Brunetti and A. M. Pastore, "Curvature of a class of indefinite globally framed f-manifolds," Bulletin Mathematique de la Societe des Sciences Mathematiques de Roumanie, vol. 51, no. 3, pp. 183-204, 2008.

[9] C. Calin, "On existence of degenerate hypersurfaces in Sasakian manifolds," Arab Journal of Mathematical Sciences, vol. 5, pp. 21-27, 1999.

[10] L. Brunetti and A. M. Pastore, "Lightlike hypersurfaces in indefiite $\mathcal{S}$-manifolds," Differential Geometry-Dynamical Systems, vol. 12, pp. 18-40, 2010.

[11] A. Bejancu, "Umbilical semi-invariant submanifolds of a Sasakian manifold," Tensor, vol. 37, pp. 203-213, 1982. 


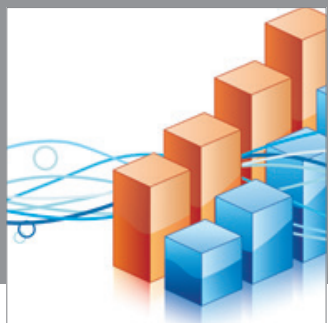

Advances in

Operations Research

mansans

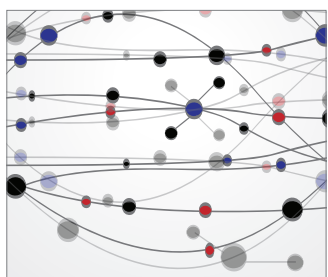

The Scientific World Journal
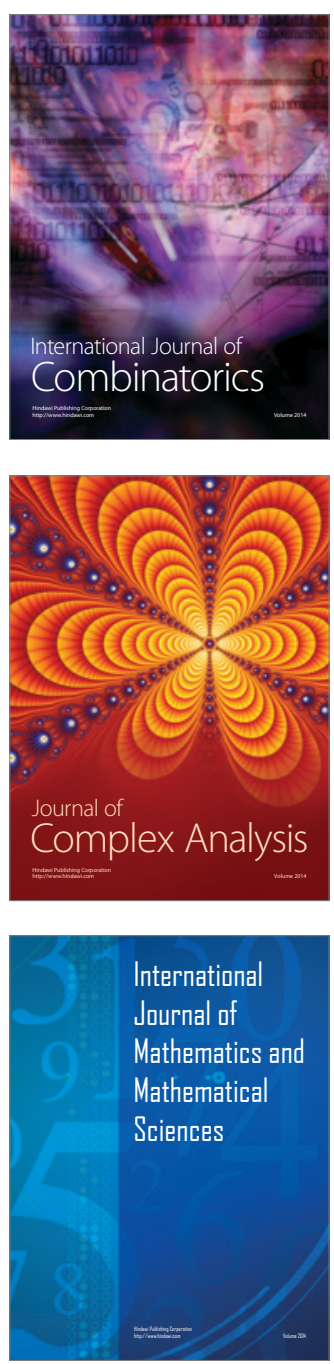
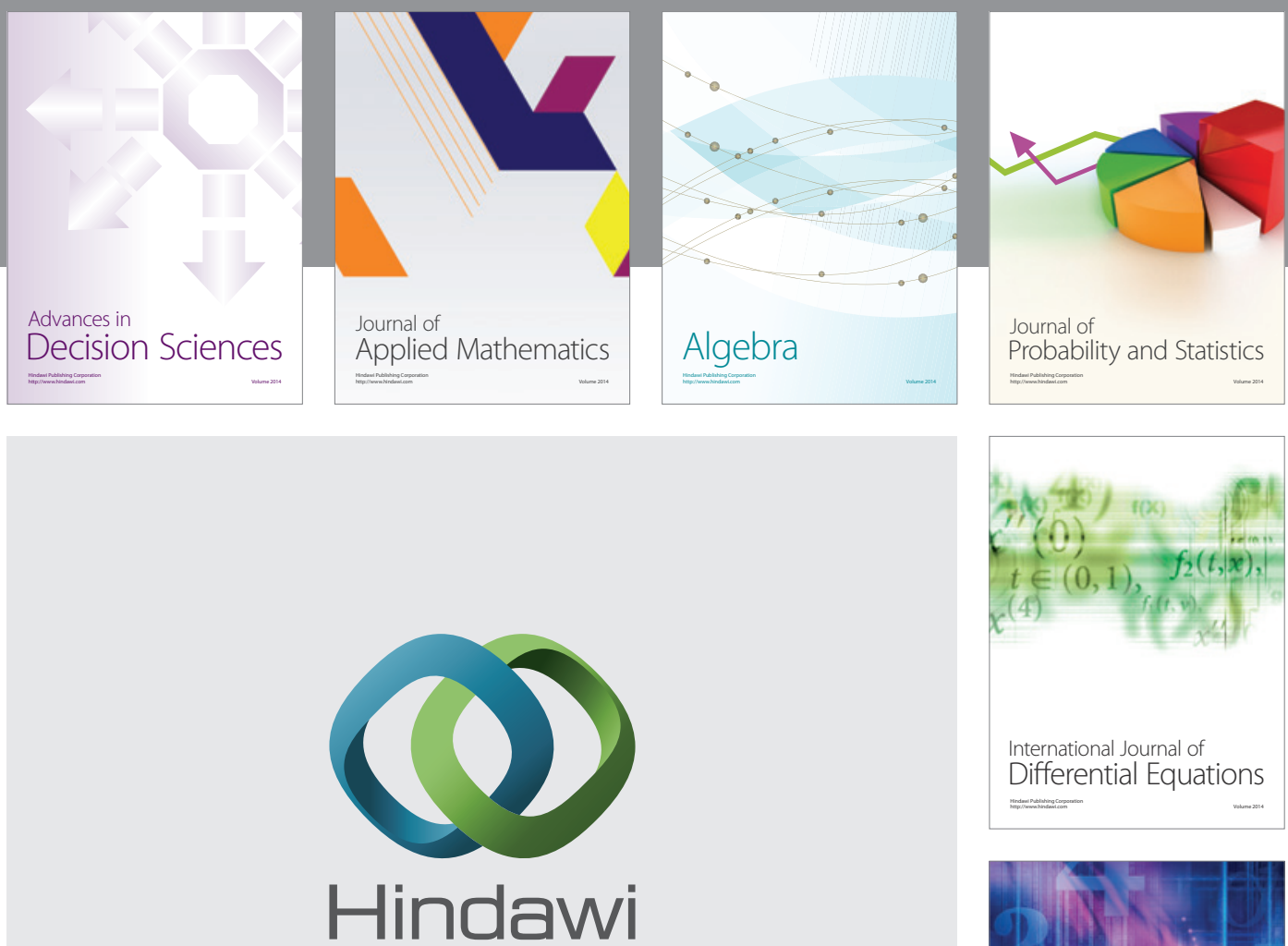

Submit your manuscripts at http://www.hindawi.com
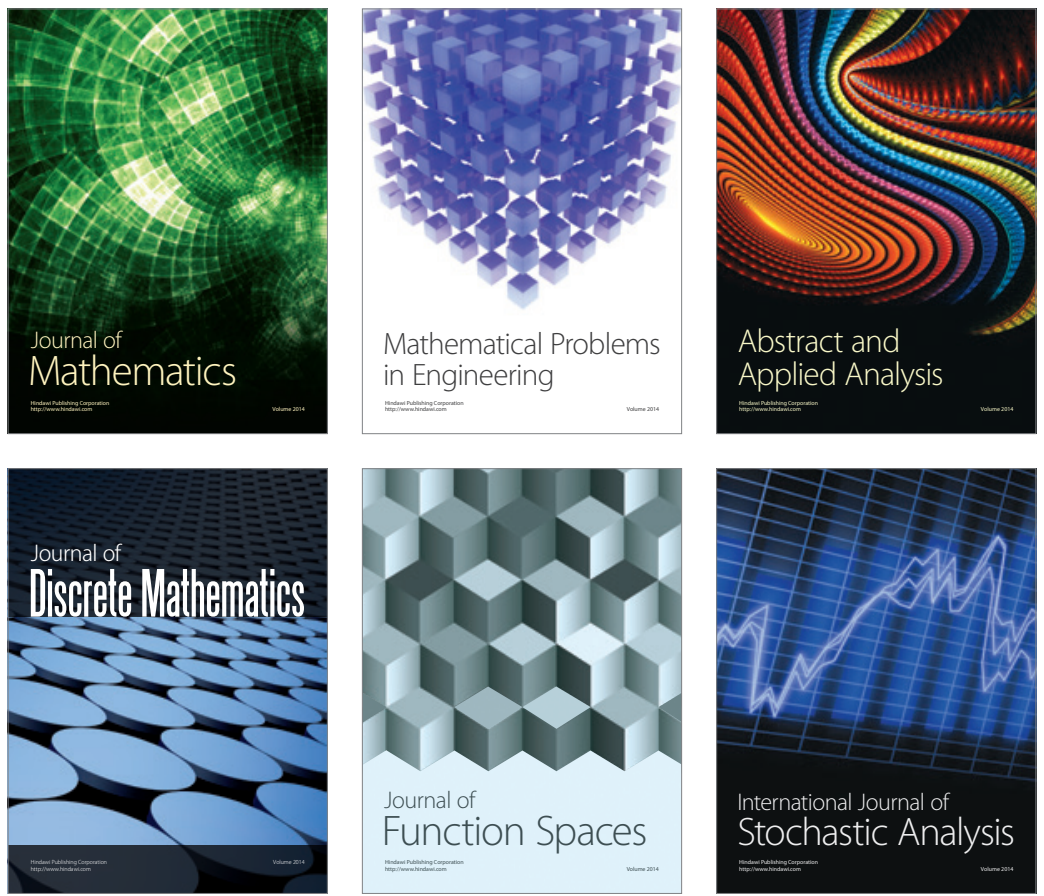

Journal of

Function Spaces

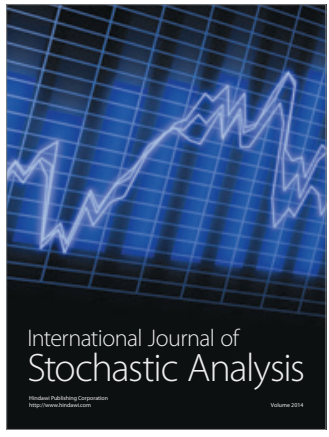

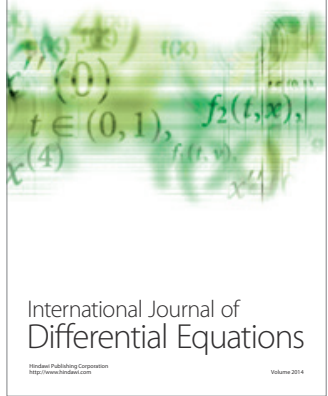
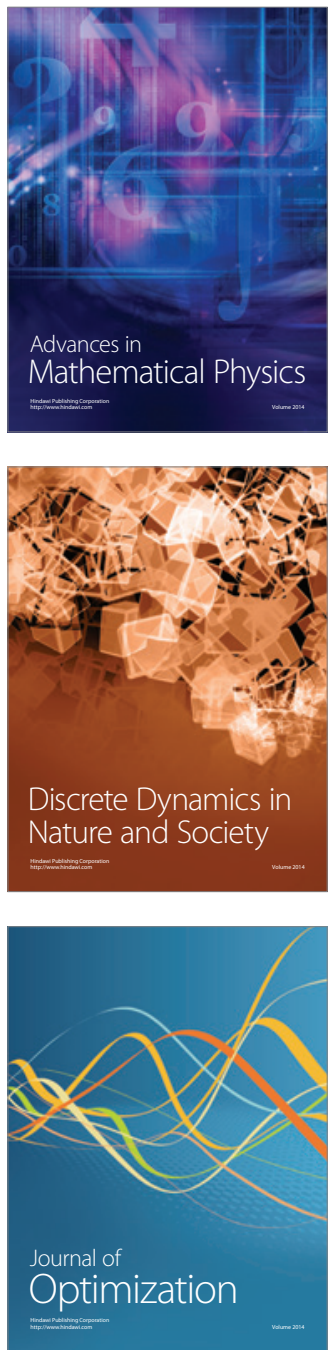